\title{
Pilot Study of the Relationship Between Heart RATE AND ECTOPY AND UNRESTRICTED VS RESTRICTED Visiting Hours In The Coronary Care UnIT
}

By Deborah Ann Schulte, RN, MSN, Lenette O. Burrell, RN, EdD, Sarah Hall Gueldner, RN, DSN, Martha H. Bramlett, RN, PhD, Barbara Fuszard, RN, PhD, Susan K. Stone, RN, MSN, CNS, and William N. Dudley, PhD. From Northeast Georgia Medical Center, Gainesville, Ga; Medical College of Georgia School of Nursing, Athens, Ga; Medical University of South Carolina, College of Nursing, Charleston, SC; Medical College of Georgia School of Nursing, Athens, Ga; Medical College of Georgia School of Nursing, Augusta, Ga; Northeast Georgia Medical Center, Gainesville, Ga; and Medical College of Georgia School of Nursing, Augusta, Ga.

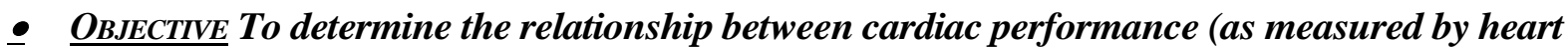
rate and ectopy) and unrestricted vs restricted visiting hours in the coronary care unit.

- DESIGN Patients were from two coronary care units. Group A had unrestricted visiting hours, and group $B$ had restricted visiting hours. Heart rate and ectopy were measured three times both in patients with unrestricted visiting hours and in those with restricted visiting hours: (1) before visitors arrived, (2) 5 minutes after visitors arrived and (3) 1 to 5 minutes after the visitors left. A total of 25 visits were analyzed.

- FINDINGS There were no significant differences in rates of premature ventricular contractions and premature atrial contractions between the two groups. Patients with unrestricted visiting hours had a significantly lower heart rate after visits than patients with restricted visits.

- ConCLUSION Consideration should be given to development of unrestricted visiting policies that promote the continuing presence and natural support of the family and significant others for patients in coronary care units. (American Journal of Critical Care. 1993;2:134-136)

The purpose of this study was to determine the relationship between cardiac performance, as measured by heart rate and ectopy - premature

ventricular contractions (PVCs) and premature atrial contractions (PACs) - and unrestricted vs restricted

Research was conducted under the auspices of the Medical College of Georgia School of Nursing/Athens. Research sites were the Northeast Georgia Medical Center in Gainesville, Ga and Gwinnett Medical Center in Lawrenceville, Ga. visiting hours in the coronary care unit (CCU). This study builds on and extends the findings of researchers who have investigated the type of visiting (unrestricted or restricted) most beneficial to CCU patients. ${ }^{\prime 2}$ There is consensus regarding the need to continue investigation into the impact of visiting policies on patient and family anxiety. ${ }^{3}$ Nurses need to identify the visiting practices most supportive to the health and well-being of patients and family members. Investigators have focused on ICU patients' responses to visits by assessing heart rate, blood pressure and the number of PVCs per minute. ${ }^{24}$ The findings consistently support permitting a family member to remain at the bedside to decrease patient anxiety and promote cardiovascular stability. 


\section{Methodology}

A two-group, time-series, quasi-experimental research design was used for this study. Subjects consisted of patients from two CCUs-one with unrestricted visiting hours (group A) and one with restricted visiting hours (group B). Restricted visiting hours were for 10 minutes at $6 \mathrm{AM}, 9 \mathrm{AM}, 1 \mathrm{PM}, 5 \mathrm{PM}$ and 8:30 PM unless an emergency arose during the visiting time, in which case visitors would be asked to leave the room. It was hypothesized that patients assigned to a unit with unrestricted visiting hours would have fewer dysrhythmias (PACs and PVCs) and a lower heart rate than patients assigned to a unit with restricted visiting hours. All subjects had admitting diagnoses of either myocardial infarction (MI) or chest pain. Subjects were required to be alert and oriented to their surroundings.

During the first 24 hours after admission, the heart rate of each subject was collected for 1 minute during two consecutive 8-hour shifts. The heart rates were averaged together to determine the patient's average (baseline) heart rate. Data collection for between-group comparisons began with visits the following morning. Heart rate and ectopy were measured three times in these patients: (1) before visitors arrived, (2) 5 minutes after visitors arrived and (3) 1 to 5 minutes after the visitors left. The variables were analyzed for differences between groups and for changes over time. Analysis was conducted using two-way analysis of variance with repeated measures. Additionally, analysis of covariance (ANCOVA) was used to control for variance in baseline measures.

\section{Findings}

The final sample for this study consisted of 25 visits to patients admitted to a CCU for a cardiac condition: 13 visits occurred in group A (unrestricted visiting policy), and 12 visits occurred in group B (restricted visiting policy).

The overall sample ranged in age from 33 to 87 years, with an overall mean of 59.92. The mean age for group A was 60.3 years and for group B, 59.5 years. This between-group difference in age was not significant at an alpha level of $P=.05$. Of the entire group, the number of subjects diagnosed with MIs was $12(48 \%)$, and the number of subjects diagnosed with chest pain was $13\left(52 \% ; X^{2}\right.$ $(1)=3.5)$. However, group A included more subjects (10) with a diagnosis of MI than group B (2). Similarly, only 3 subjects in group A were diagnosed with chest pain, compared with 10 subjects in group B. Thus, groups A and B were significant ly different in terms of diagnosis as determined by chi-square analysis $\left(X^{2}=9.077, d f=1, P>.01\right)$. Group A had significantly more subjects with the more severe diagnosis of MI, whereas group B had significantly more subjects with the milder diagnosis of chest pain.

Data analysis revealed no significant differences in rates of PVCs and PACs between the two groups. However, significant differences between group A and group B were revealed by ANCOVA over time $(\mathrm{F}(2,46)=3.75, P=.030)$. Patients with unrestricted visits (group A) experienced significant decreases in heart rate after visits, whereas patients with restricted visits (group B) displayed no such changes (see Figure).

\section{Discussion}

Cardiac performance is an important determinant of expected patient outcomes, serving as a measure of the efficacy of treatment, as well as a useful means of determining prognosis. Exploration of beneficial forms of visiting that can potentially relieve and/or reduce the patient's anxiety, as well as reduce adverse effects on heart rate or ectopy, provide one means of improving patient outcomes. The data suggest that unrestricted visiting may contribute to decreased anxiety levels in coronary care patients, as indicated by decreased heart rates after visits.

In evaluating these results, several possible confounding variables must be considered. It was not possible to sample a single unit; performing the study in two units, however, is a possible source of error. The sample size was small, with only 13 subjects in group A and 12 subjects in group B. A larger sample size might have demonstrated additional findings. Only the first visit of each patient was used. Recurring visits could have had greater impact on anxiety, and therefore heart rate and ectopy, over time. Thus, a study of responses to subsequent visiting times might have yielded greater or different results.

No attempt was made to control for the time medications were distributed or the types of medications administered. Types and frequency of medications were not included in data collection. Because the drugs administered to CCU patients are often specifically for dysrhythmias and heart rate, these medications could have had significant impact on the study results. In addition, it was not possible to control the activities that occurred immediately after the visitors left or to control for the quality of interactions during visits. These factors could have had various effects on patient anxiety and therefore on the study results. 


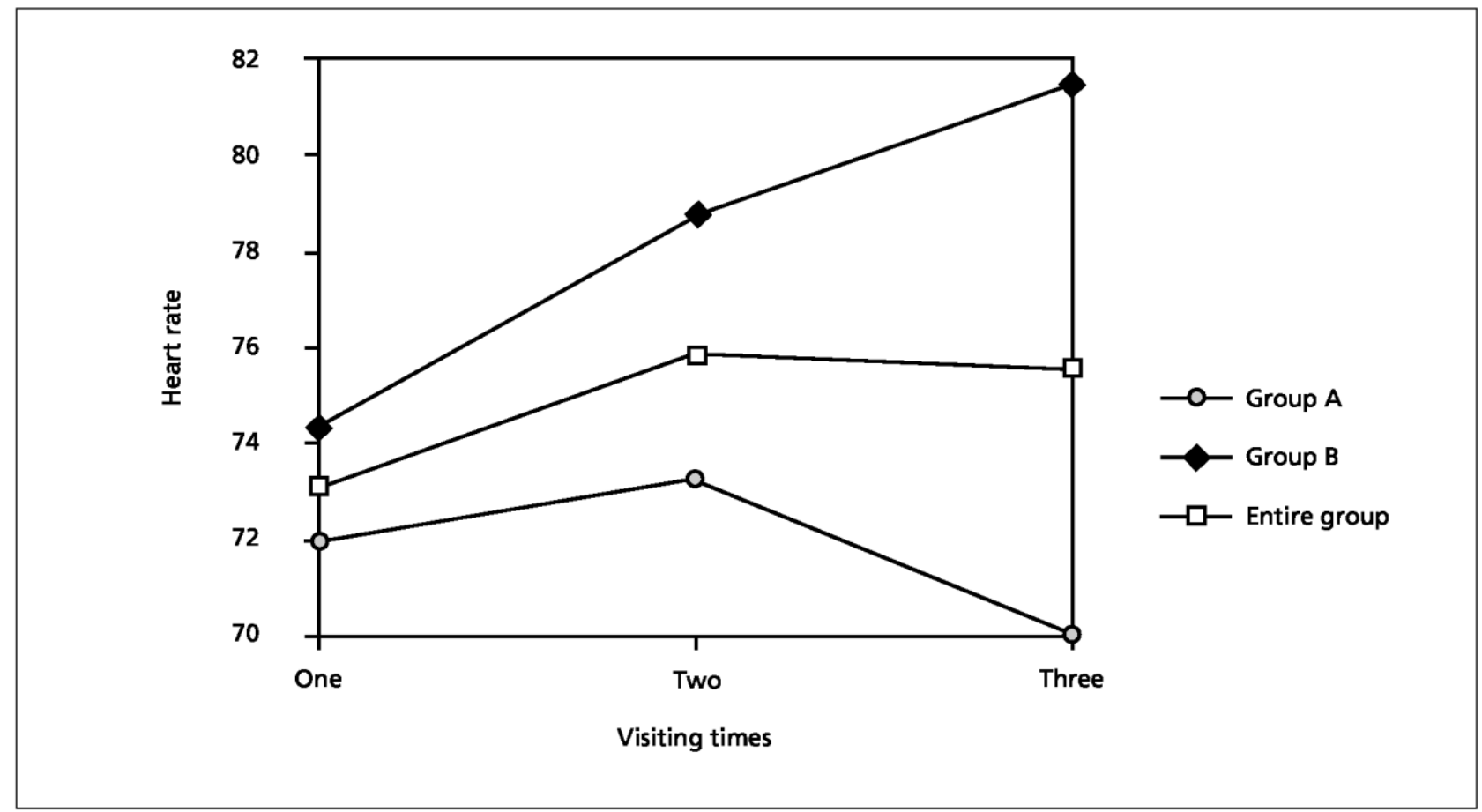

Group A included a significantly higher number of subjects diagnosed with MI than group B. Therefore, group A may have been composed of subjects with a higher acuity of illness who would be expected to exhibit more dysrhythmias than group B. Consequently, they would have required more antidysrhythmic medications. If this were the case, group A may have had a lower rate of ectopy than group B as a result of antidysrhythmics, rather than the postulated effect of unrestricted visiting hours.

\section{Conclusions}

A larger sample size is needed in future studies of this type and groups should comprise subjects with diagnoses of approximately equivalent acuity. More over, activities of patients during visits and for 1 to 5 minutes after visitors leave should be recorded and controlled when possible. Data should also be studied regarding types and scheduling of medications. Such information may provide more complete insight into the potential effects of medications on heart rate and ectopy during visits.

An important implication for critical care management has been revealed in these findings. In analyzing and comparing the effects of unrestricted vs restricted visiting in this study, a subtle but important difference in cardiac performance was detected.
Although there were no significant differences between the groups relative to ectopy, the mean heart rate for patients with unrestricted visits was significantly lower after visits than the mean heart rate of patients with restricted visits. By lowering the heart rate of the patients admitted into a CCU by the type of visiting hours, it may be possible to decrease morbidity as well as mortality. Although this finding needs to be confirmed through replicated studies, it seems clear that consideration should be given to the development of unrestricted visiting policies that promote the continuing presence and natural support of the family and significant others for patients in the CCU.

\section{REFERENCES}

1. Thomas S, Lynch J, Mills M. Psychosocial influences on heart rhythm in the coronary-care unit. Heart Lung. 1975;4:746-750.

2. Brown A. Effect of family visits on the blood pressure and heart rate of patients in the coronary-care unit. Heart Lung. 1976; 5:291-296.

3. Kirchhoff K, Hansen C, Evans P, Fullmer N. Open visiting in the ICU: a debate. Dimensions of Critical Care Nursing. 1985; 4:296-304 